\title{
Second-harmonic imaging of ferroelectric domain walls.
}

Bozhevolnyi, Sergey I.; Hvam, Jørn Märcher; Pedersen, Kjeld; Laurell, Fredrik; Karlsson, Håkan; Skettrup, Torben; Belmonte, Michele

Published in:

Applied Physics Letters

Link to article, DOI:

$10.1063 / 1.122291$

Publication date:

1998

Document Version

Publisher's PDF, also known as Version of record

Link back to DTU Orbit

Citation (APA):

Bozhevolnyi, S. I., Hvam, J. M., Pedersen, K., Laurell, F., Karlsson, H., Skettrup, T., \& Belmonte, M. (1998). Second-harmonic imaging of ferroelectric domain walls. Applied Physics Letters, 73(13), 1814-1816.

https://doi.org/10.1063/1.122291

\section{General rights}

Copyright and moral rights for the publications made accessible in the public portal are retained by the authors and/or other copyright owners and it is a condition of accessing publications that users recognise and abide by the legal requirements associated with these rights.

- Users may download and print one copy of any publication from the public portal for the purpose of private study or research.

- You may not further distribute the material or use it for any profit-making activity or commercial gain

- You may freely distribute the URL identifying the publication in the public portal

If you believe that this document breaches copyright please contact us providing details, and we will remove access to the work immediately and investigate your claim. 


\title{
Second-harmonic imaging of ferroelectric domain walls
}

\author{
Sergey I. Bozhevolnyia) and Jørn M. Hvam \\ Mikroelektronik Centret, Technical University of Denmark, DK-2800 Lyngby, Denmark \\ Kjeld Pedersen \\ Institute of Physics, Aalborg University, DK-9220 Aalborg, Denmark \\ Fredrik Laurell \\ Department of Physics, Royal Institute of Technology, 10044 Stockholm, Sweden \\ Håkan Karlsson \\ Institute of Optical Research, 10044 Stockholm, Sweden
}

Torben Skettrup and Michele Belmonte

Department of Physics, Technical University of Denmark, DK-2800 Lyngby, Denmark

(Received 7 May 1998; accepted for publication 28 July 1998)

Domain walls in periodically poled ferroelectric $\mathrm{KTiOPO}_{4}$ and $\mathrm{LiNbO}_{3}$ crystals are observed by making use of second-harmonic $(\mathrm{SH})$ generation enhancement in the transition regions between neighboring domains. SH images of domain walls obtained with various samples for different polarization configurations are presented. The SH generation enhancement is found especially pronounced for the polarization of the SH radiation being perpendicular to the domain walls. The origin and selection rules for the contrast in SH images of domain walls are discussed. The results obtained suggest that the domain walls produce a deteriorating effect on $\mathrm{SH}$ generation by quasiphase matching. (C) 1998 American Institute of Physics. [S0003-6951(98)02039-7]

Second-harmonic generation (SHG) involves, in general, interaction between three optical waves, and therefore, is extremely sensitive to the symmetry of materials. ${ }^{1}$ In particular, SHG has been extensively used as a nondestructive and noncontact probe of surfaces and interfaces. ${ }^{2}$ SHG can be further employed to image variations in nonlinear susceptibilities of the inspected material. Thus, SHG microscopy has been successfully applied to visualization of periodically poled ferroelectric domains, ${ }^{3}$ domain structures in epitaxially grown magnetic garnet films, ${ }^{4}$ and polar orientational distribution in thin polymer films. ${ }^{5}$ Nondestructive imaging of $180^{\circ}$ (antiparallel) domains, ${ }^{2}$ which are optically equivalent, is rather important from the point of view of both basic research and various applications, e.g., for characterization of frequency conversion devices utilizing quasiphase matching (QPM) by periodically poled domains. ${ }^{6}$ The transition regions between neighboring domains, i.e., domain walls, is perhaps an even more interesting object for SHG microscopy: nonlinear optical properties of domain walls are largely unknown but progressively important (e.g., for QPM-based devices) with the decrease of distances between the walls (e.g., the spatial period of QPM structures). $180^{\circ}$ domain walls in a $\mathrm{LiTaO}_{3}$ single crystal have been imaged with linear optical methods, viz., by using unpolarized light ${ }^{7}$ and polarization sensitive techniques, ${ }^{7,8}$ suggesting that light is scattered by the walls and its polarization is modified due to strain-induced birefringence. $^{7,8}$

In this letter, we demonstrate that the domain walls in periodically poled ferroelectric $\mathrm{KTiOPO}_{4}(\mathrm{KTP})$ and $\mathrm{LiNbO}_{3}$ (LN) crystals can be imaged with SHG microscopy, and consider the origin of the selection rules in optical secondharmonic (SH) images. The experimental setup (the SHG

${ }^{a)}$ Corresponding author. Electronic mail: sbo@mic.dtu.dk microscope $\mathrm{e}^{3}$ ) used to image $180^{\circ}$ domain walls represents a projection microscope in transmission with a powerful laser as a light source and a charge-coupled device (CCD) camera as a sensitive image detector [Fig. 1(a)]. The linearly polarized light beam from a mode-locked Ti-sapphire laser $\left(\lambda_{\omega}\right.$ $\approx 800 \mathrm{~nm}, P_{\omega}^{\mathrm{av}} \approx 300 \mathrm{~mW}$, repetition rate $f \approx 80 \mathrm{MHz}$, pulse duration $\tau \sim 200 \mathrm{fs}$ ) is focused with a $3 \times$ micro-objective (numerical aperture, $\mathrm{NA}=0.11$ ) on a periodically poled crystal, whose $Z$ axis is oriented parallel to the incident beam (Fig. 1). The transmitted pump radiation, i.e., the firstharmonic $(\mathrm{FH})$, and the $\mathrm{SH}$ waves generated in the crystal are collected by a $20 \times$ micro-objective $(\mathrm{NA}=0.4)$ to form a FH or SH image (depending on the choice of filter) with the help of a CCD camera connected with a TV monitor and a computer (to store images). The polarizations of the pump beam and the detected SH (or FH) waves can be adjusted with the help of a $\lambda / 2$ plate and a polarizer (Fig. 1).

Two KTP samples, N1 and N2, with periodic $(\Lambda$ $=9 \mu \mathrm{m}) 180^{\circ}$ domains were prepared by following a re-

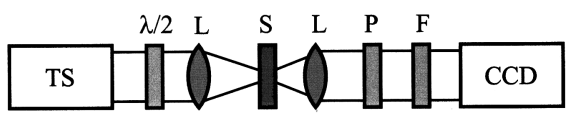

(a) (b)

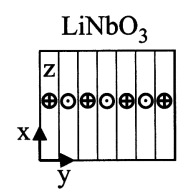

(c)

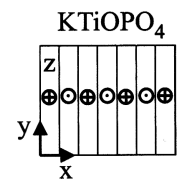

FIG. 1. Experimental setup (a) for second-harmonic imaging of periodically poled ferroelectrics [(b) and (c)]. TS, Ti-sapphire laser; $\lambda / 2$; wave plate; $\mathrm{L}$, micro-objectives; S, sample; P, polarizer; F, IR-absorption filter; and CCD, camera. 
cently developed technique. ${ }^{9}$ KTP samples $\left(9 \times 4 \times 1 \mathrm{~mm}^{3}\right)$ were cut from a flux-grown crystal, and their $z^{-}$faces were ion exchanged in a pure $\mathrm{RbNO}_{3}$ melt at $350{ }^{\circ} \mathrm{C}$ during $3.5 \mathrm{~h}$. The ion exchange procedure results in a reduction of the ionic conductivity of the crystal. The samples were then patterned with a periodic photoresist on the ion exchanged side and coated with aluminum on the same (sample N2) or opposite (sample N1) side. Domain reversal under the regions free from photoresist was obtained by applying (3-6) short electric pulses of $2 \mathrm{kV}$ to the samples using a nearly saturated solution of $\mathrm{KCl}$ as liquid electrodes. When used for SHG both samples exhibited characteristics similar to those reported previously. ${ }^{9}$

Two LN samples, N3 and N4, with periodic antiparallel domains were fabricated. $Z$-cut $\mathrm{LN}$ wafers were polished down to the thickness of $220 \mu \mathrm{m}$, and $180^{\circ}$ domains were then produced by means of electric-field poling. ${ }^{10}$ Two types of Al gratings were fabricated on the $z^{+}$crystal face $(20-\mu \mathrm{m}$ period for sample N3, and a four-sectioned grating with 8-, $12-, 16-$, and $20-\mu \mathrm{m}$ periods for sample N4). The $z^{-}$crystal face was covered with an $\mathrm{Al}$ layer, and a couple of 0.2-s-long electric-field pulses with the strength of $\sim 25 \mathrm{kV} / \mathrm{mm}$ were applied to the electrodes. The periodically poled domain pattern formed in this way penetrated the complete thickness of the crystals. Sample N3 was then imaged with the SHG microscope without further processing, whereas sample N4 was first glued to a fused quartz substrate and optically polished.

Antiparallel domains are equivalent with respect to linear optical properties and even with respect to nonlinear intensity characteristics (e.g., SHG efficiency). It means that the domains should not be visible in the SHG microscope, if there is no reference $\mathrm{SH}$ wave to reveal the difference in the SH phase via the intensity contrast. ${ }^{3,11}$ The domain walls being transition regions between oppositely oriented domains were expected to exhibit (partial) cancellation of nonlinear properties, and thereby to become visible as dark lines on a homogeneous background of the SH radiation from the domains. In fact, the domain walls if visible appeared as bright lines for all samples, periods of domain inversion, and polarization configurations (Figs. 2-4). Here, taking into account

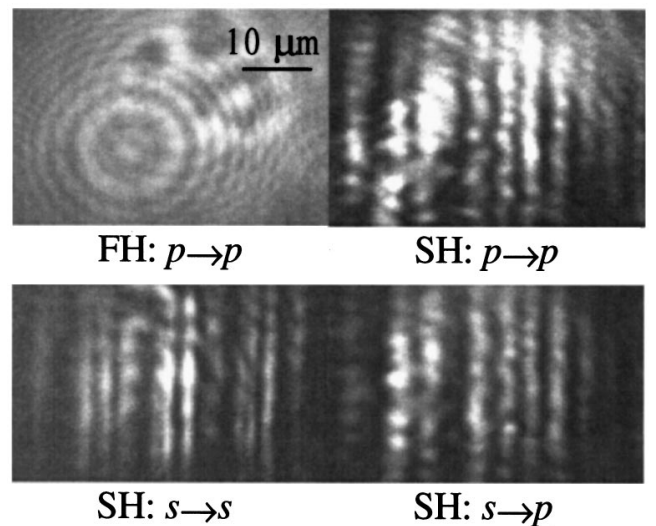

FIG. 2. First- (FH) and second-harmonic (SH) images of KTP sample N1 with the period of $9 \mu \mathrm{m}$. The acquisition time when imaging with the SHG channel $s \rightarrow p$ is two times and the time with $s \rightarrow s$ is four times larger than the time used with the channel $p \rightarrow p$. No periodic structure was observed on SH images taken with the SHG channel $p \rightarrow s$. Similar images were obtained with sample N2. inserted bar is $10 \mu \mathrm{m}$.
tained with sample N2.
Downloaded 20 Jan 2010 to 192.38.67.112. Redistribution subject to AlP license or copy
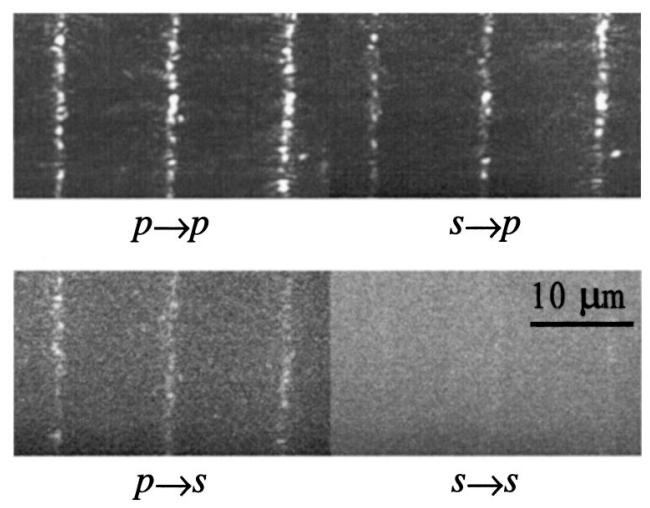

FIG. 3. High-resolution second-harmonic images of LN sample N3 with the period of $20 \mu \mathrm{m}$ for different polarization configurations. The acquisition time when imaging with the SHG channel $p \rightarrow p$ is four times smaller than the time used for other channels.

that the domain walls represent interfaces, we denoted the polarization parallel to the domain walls as $s$ polarization and the perpendicular one as $p$ polarization. There were several interesting features observed in the course of the experiment conducted: (i) the domain walls were not seen on FH images but only on SH images (Fig. 2); (ii) the domain walls appeared with different brightness for different polarization configurations (Figs. 2 and 3); (iii) the polarization configurations, for which the domain walls did appear on the $\mathrm{SH}$ images, were different for KTP and LN samples (cf. Figs. 2 and 3); (vi) for all samples, the domain walls were the brightest for the $p \rightarrow p$ configuration (Figs. 2 and 3); and (v) the SH signal distribution along the domain wall was largely the same for different polarization configurations (cf. $p \rightarrow p$ and $s \rightarrow p$ configurations in Figs. 2 and 3).

We believe that the origin of the SH enhancement observed in the domain walls can be ascribed to the effect of field enhancement and depolarization near small scatterers. ${ }^{12}$ Actually, the homogeneous background of the $\mathrm{SH}$ radiation from the domains should be very weak given the small coherence lengths and large thickness of the samples $(\sim 3$ and $220 \mu \mathrm{m}$ for $\mathrm{LN}, \sim 10 \mu \mathrm{m}$ and $1 \mathrm{~mm}$ for KTP, respectively). On the other hand, any enhancement of a FH field would produce a quadratic enhancement in the SH field. This feature is illustrated by the FH and SH images (Fig. 5) representing a small scatterer found in sample N3 outside the region with $180^{\circ}$ domains. Moreover, SH waves generated by random (in shapes and sizes) scatterers would inevitably

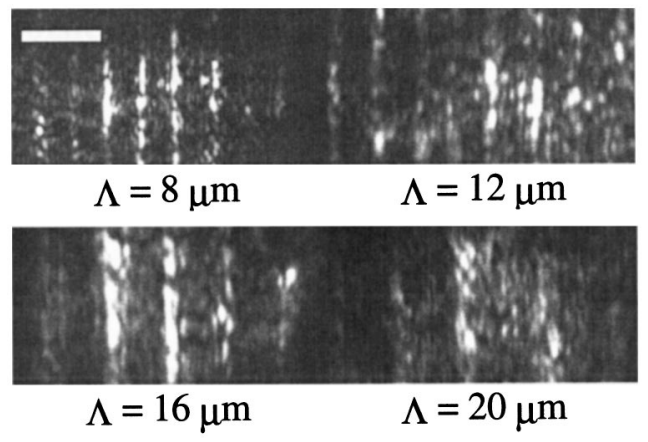

FIG. 4. Second-harmonic images of LN sample N4 for regions with different periods $\Lambda$ for the $p \rightarrow p$ polarization configuration. The length of the

AIP license or copyright; see http://apl.aip.org/apl/copyright.jsp 

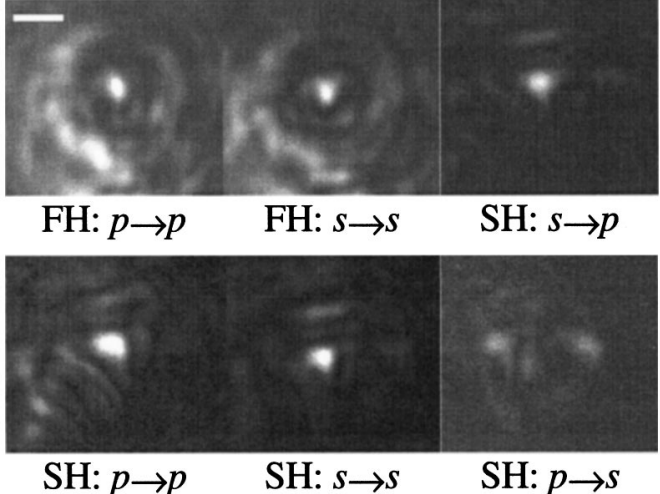

FIG. 5. First- $(\mathrm{FH})$ and second-harmonic (SH) images of a small scatterer found in LN sample N3 outside the region with antiparallel domains. The length of the inserted bar is $10 \mu \mathrm{m}$. The acquisition time used to obtain the $\mathrm{SH}$ images is the same for all polarization configurations.

be dephased, thereby decreasing the effect of destructive (due to phase mismatch) coherent interference. The fact that the $\mathrm{SH}$ images of domain walls are inhomogeneous along the walls and similar for different polarizations (Figs. 2 and 3) supports our conjecture. These scatterers are likely to be related to strain-induced defects (dislocations) as suggested for $180^{\circ}$ domains in $\mathrm{LiTaO}_{3}$ crystals. ${ }^{7,8}$ Stress and associated defects can also be introduced by polishing, and $\mathrm{SH}$-related scattering inside the domains is clearly seen on the images (Fig. 4) obtained with sample N4 that has been polished.

Let us now consider the selection rules observed in our experiments. The leading polarization terms that are responsible for the detected SH signal can be written as follows: ${ }^{1}$

$$
\begin{aligned}
& P_{x}^{2 \omega}=2 d_{15} E_{x}^{\omega} E_{z}^{\omega}+2 d_{16} E_{x}^{\omega} E_{y}^{\omega}, \\
& P_{y}^{2 \omega}=d_{22}\left[\left(E_{y}^{\omega}\right)^{2}-\left(E_{x}^{\omega}\right)^{2}\right]+2 d_{24} E_{y}^{\omega} E_{z}^{\omega},
\end{aligned}
$$

where $d_{i k}$ are nonlinear susceptibilities $\left(d_{24}=d_{15}\right.$ and $d_{16}$ $=-d_{22}$ for $\mathrm{LN}$, and $d_{16}=d_{22}=0$ for $\left.\mathrm{KTP}\right)$, and $E_{k}^{\omega}(k$ $=x, y, z)$ are the FH field amplitude components (Fig. 1). It is immediately seen that for a plane linearly polarized (along the $x$ or $y$ axis) FH wave, only one polarization of a SH wave is expected and only for $\mathrm{LN}: P_{y}^{2 \omega}=d_{22}\left[\left(E_{y}^{\omega}\right)^{2}-\left(E_{x}^{\omega}\right)^{2}\right]$. Consequently, the images of the domain walls in KTP (Fig. 2) and (for the $p \rightarrow s$ configuration) LN (Fig. 3) can be accounted for only by the effect of depolarization of the $\mathrm{FH}$ field. Since the scattered field contains all components in the near-field region (where it is also enhanced), ${ }^{12}$ depolarization effects are expected in SHG from scatterers (domain walls) and, for example, clearly seen on the images obtained with the small scatterer (Fig. 5). Judging from these images, SHG channels $s \rightarrow s$ and $s \rightarrow p$ should be equally efficient and thereby produce similar images of the domain walls in LN. However, the domain walls are not seen for the $s \rightarrow s$ configuration (Fig. 3). Furthermore, assuming the depolarization to be a relatively small effect (scatterers are sufficiently weak, since the walls are not seen on the FH images), one would expect the $\mathrm{SH}$ images of the domain walls in KTP to be equally bright for the following channels [see Eqs. (1) and (2)]: $p \rightarrow p$ and $s \rightarrow s, s \rightarrow p$ and $p \rightarrow s$. Again, these prognoses turn out being wrong (Fig. 2). The overall conclusion is that the $\mathrm{SH}$ radiation polarized perpendicular to the domain walls is exceptionally strong. This means, in turn, that the scatterers by being arranged along the domain walls acDownloaded 20 Jan 2010 to 192.38.67.112. Redistribution subject quired additional symmetry in the nonlinear response. Actually, if the interface density of random defects (along the wall plane) is large, one should expect to find additional selection rules for $\mathrm{SHG}$, i.e., those existing for the interface SHG in the case of isotropic media. These rules only allow $p$-polarized $\mathrm{SH}$ radiation. ${ }^{2}$ We believe that, in our case, the same rules can be applied if only to some extent depending on the strength and interface density of wall defects.

The experiments conducted with different samples strongly indicate that, as far as SHG is concerned, $180^{\circ}$ ferroelectric domain walls can be considered as being composed of microdefects with the effect of partial depolarization and imposing interface selection rules. The most important consequence is that the effective nonlinearity in QPM devices is expected to decrease with the decrease of the inversion period. Indeed, in QPM devices, the pump FH and generated $\mathrm{SH}$ waves propagate perpendicular to the walls, ${ }^{6}$ and, therefore, SHG taking place in the wall region is ineffective (because it is forbidden by the interface selection rules) as compared with that in the domain region. It is our experience with QPM structures based on KTP crystals, that the effective nonlinearity (deduced from the measurements of SHG efficiency) for QPM structures with periods down to $\Lambda=9 \mu \mathrm{m}\left(d_{33}^{\mathrm{eff}}=14-17 \mathrm{pm} / \mathrm{V}\right)$ is close to the ideal one $(18.5$ $\mathrm{pm} / \mathrm{V})$, whereas the nonlinearity for denser gratings is reduced: $d_{33}^{\mathrm{eff}}=5-10 \mathrm{pm} / \mathrm{V}$ for $\Lambda=4 \mu \mathrm{m}$ and becomes even lower than that for the gratings with smaller periods used for UV light generation. These observations support our hypothesis about the domain walls, and even fit quantitatively if the thickness of the domain walls is taken to be $\sim 1 \mu \mathrm{m}$. Such a value has been reported for the domain walls in ferroelectric $\mathrm{LiTaO}_{3}$ crystals measured with a linear polarization sensitive near-field microscope. ${ }^{8}$

In summary, using second-harmonic imaging we have visualized $180^{\circ}$ domain walls in two different ferroelectrics and investigated the origin of the imaging mechanism and the corresponding selection rules. It is shown that, with respect to SHG, the domain walls can be treated as a nearly isotropic nonlinear interface layer with depolarization properties. The technique used can be further exploited to investigate nonlinear properties of the domain walls fabricated in other crystals, and also as a function of fabrication and geometrical parameters, e.g., as a function of the inversion period.

${ }^{1}$ R. W. Boyd, Nonlinear Optics (Academic, London, 1992).

${ }^{2}$ T. F. Heinz, in Nonlinear Surface Electromagnetic Phenomena, edited by H. Ponath and G. Stegeman (Elsevier, Amsterdam, 1991), p. 353.

${ }^{3}$ S. Kurimura and Y. Uesu, J. Appl. Phys. 81, 389 (1997); Y. Uesu, S. Kurimura, and Y. Yamamoto, Appl. Phys. Lett. 66, 2165 (1995).

${ }^{4}$ V. Kirilyuk, A. Kirilyuk, and Th. Rasing, Appl. Phys. Lett. 70, 2306 (1997).

${ }^{5}$ J. Vidra and M. Eich, Appl. Phys. Lett. 72, 275 (1998).

${ }^{6}$ M. M. Fejer, G. A. Magel, D. H. Jundt, and R. L. Byer, IEEE J. Quantum Electron. 28, 2631 (1992).

${ }^{7}$ V. Gopalan and M. C. Gupta, J. Appl. Phys. 80, 6099 (1996).

${ }^{8}$ T. J. Yang, U. Mohideen, and M. C. Gupta, Appl. Phys. Lett. 71, 1960 (1997).

${ }^{9}$ H. Karlsson and F. Laurell, Appl. Phys. Lett. 71, 3474 (1997).

${ }^{10}$ L. E. Myers, R. C. Eckardt, M. M. Fejer, R. L. Byer, W. R. Bosenberg, and J. W. Pierce, J. Opt. Soc. Am. B 12, 2102 (1995).

${ }^{11}$ F. Laurell, M. G. Roelofs, W. Bindloss, H. Hsiung, A. Suna, and J. D. Bierlein, J. Appl. Phys. 71, 4664 (1992).

${ }^{12}$ J. D. Jackson, Classical Electrodynamics (Wiley, New York, 1975). AIP license or copyright; see http://apl.aip.org/apl/copyright.jsp 